Supporting Information

\title{
Outdoor personal thermal management with simultaneous electricity generation
}

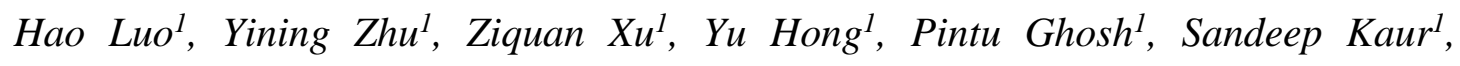
Mingbang $\mathrm{Wu}^{2}$, Chenying Yang ${ }^{1}$, Min Qiu $^{3,4, *}$ and Qiang $\mathrm{Li}^{1, *}$

${ }^{1}$ State Key Laboratory of Modern Optical Instrumentation, College of Optical Science and Engineering, Zhejiang University, Hangzhou 310027, China

${ }^{2}$ School of Materials Science and Engineering, Zhejiang Sci-Tech University, 928

Second Avenue, Xiasha Higher Education Park, Hangzhou 310018, China

${ }^{3}$ Key Laboratory of 3D Micro/Nano Fabrication and Characterization of Zhejiang

Province, School of Engineering, Westlake University, 18 Shilongshan Road, Hangzhou 310024, Zhejiang Province, China

${ }^{4}$ Institute of Advanced Technology, Westlake Institute for Advanced Study, 18 Shilongshan Road, Hangzhou 310024, Zhejiang Province, China 


\section{Supplement 1. Characterization methods}

Thermal measurement. All thermal measurements are performed with thermocouples (Omega, SA-1K) and a Keithley 2700 multimeter is used to acquire the real-time temperature. The optical images are shot with Fujifilm X-E3 camera and XF50 f/2 lens. The test samples $(10 \mathrm{~cm} \times 10 \mathrm{~cm})$ are fixed on the skin simulators with a sample fixture. The thickness of the tested black cotton is $1 \mathrm{~mm}$ and the thickness of the tested white cotton is $500 \mu \mathrm{m}$. The skin simulator is made up of a $10 \mathrm{~cm} \times 10 \mathrm{~cm}$ rubber heater $(100 \mathrm{~W}$ $\mathrm{m}^{-2}$ input power) adhered with skin tape (3M micropore 1533-1, optical properties in Figure S5). A $10 \mathrm{~cm} \times 10 \mathrm{~cm} \times 10 \mathrm{~cm}$ foam (expanded polystyrene, EPS) is used to hold the skin simulator which acts as a heat insulator. The whole thermal chamber is a foam box adhered with aluminum foils to reduce heat transfer through conduction and radiation from the ambient. The thermocouples are attached to the upper surfaces of the skin simulator. Thermocouple for ambient temperature measurement is attached on the side wall of the foam beneath the skin simulator and sheltered from sunlight. During thermal measurement, the thermal chamber is placed facing the clear sky without any tilt and exposed to wind flow. A pyranometer and an anemometer are used to record real time solar irradiance power density and wind speed, respectively. Both daytime and nighttime tests were conducted on March $4^{\text {th }}$ in Hangzhou, China.

Thermal electricity measurement. A $4.3 \mathrm{~mm}$ thick commercial thermoelectric generator (TEG, model: TGM-263-1.4-1.8) covered by the Janus textile is directly put on the healthy human's skin (male, 25-year-old). Thermocouples are attached on the skin and the upper side of the TEG for measuring the temperature difference. The output thermal electricity voltages and the temperature are recorded by the Keithley 2700 multimeter. A pyranometer and an anemometer are used to record real time solar irradiance power density and wind speed, respectively. The maximum output power density is calculated by $P_{\max }=U^{2} / 4 R / A$, where $\mathrm{U}$ is the output voltage, $\mathrm{R}$ is the measured resistance of the thermoelectric module and is $10 \Omega$, the area size A is 4.8 $\mathrm{cm} \times 5.2 \mathrm{~cm}$. Both daytime and nighttime tests were conducted on March $22^{\text {nd }}$ in Hangzhou, China. 
Water vapor transmission rate measurement. The test was performed using ASTM E96 with modification. Petri dishes filled with $10 \mathrm{ml}$ distilled water were sealed by the textile samples using rubber bands. These sealed dishes were then put into an environmental chamber whose temperature was kept at $30^{\circ} \mathrm{C}$ and relative humidity at $30 \%$. The dishes were weighed periodically with an electronic balance (OHAUS, AR1502CN). The water vapor transmission rates were calculated from the mass loss, which was equal to the mass of evaporated water.

Water contact angle measurement. The static water contact angles were measured by a DropMeter A-200 contact angle system (MAIST Vision Inspection \& Measurement Co. Ltd., China) in the ambient environment to evaluate the wettability of the textile.

\section{Supplement 2. Heat transfer model analysis}

A one-dimensional heat transfer model is used to determine the total heat dissipation rate of human body wearing textile of different optical properties. In this model, sunlight illumination, thermal radiation, conduction, and convection are included to simulate the heat dissipation from the body to ambient air (Figure S1).

For the textile covered skin, the energy balance at skin surface:

$$
q=\left(1-r_{i, M I R}\right) \cdot q_{\text {rad }, s}-q_{\text {rad }, i}-\frac{\tau_{\text {sun }, t} \cdot \alpha_{s} \cdot q_{\text {sun }}}{1-r_{\text {sun }, s} \cdot r_{\text {sun }, t}}+q_{\text {cond }, a}
$$

Energy balance at textile outer surface:

$$
q=-q_{a t m}+q_{\text {rad }, o}+q_{c o n v}-\left[\left(1-r_{\text {sun }, t}\right)-\frac{\tau_{\text {sun }, t}^{2} \cdot r_{\text {sun }, s}}{1-r_{\text {sun }, s} \cdot r_{\text {sun }, t}}\right] \cdot q_{\text {sun }}
$$

The temperature profile within the textile:

$$
\begin{aligned}
& T_{o}=\frac{t_{t}}{2 k_{t}}\left\{q_{\text {rad }, i}+q_{\text {rad }, o}-\left(1-r_{i, M I R}\right) \cdot q_{\text {rad }, s}-q_{\text {atm }}-\right. \\
& \left.\left[\left(1-r_{\text {sun }, t}\right)-\frac{\tau_{\text {sun }, t}{ }^{2} \cdot r_{\text {sun }, s}}{1-r_{\text {sun }, s} \cdot r_{\text {sun }, t}}-\frac{\tau_{\text {sun }, t} \cdot \alpha_{s}}{1-r_{\text {sun }, s} \cdot r_{\text {sun }, t}}\right] \cdot q_{\text {sun }}\right\}-\frac{k_{a} t_{t}}{k_{t} t_{a}} \cdot\left(T_{s}-T_{i}\right)+T_{i}
\end{aligned}
$$


where $q$ is the body heat generation rate per unit area, $\alpha$ and $q_{\text {sun }}$ is sunlight absorptivity of the textile and solar irradiation power density, $q_{\text {rad,atm }}$ is the absorbed radiation heat flux from ambient air, $q_{\text {rad }, i}$ is the radiation heat flux from the inner surface of textile, $q_{r a d, s}$ is the related to the radiation heat flux from skin, $q_{\text {cond,a }}$ is the conductive heat flux in the air gap between skin and textile, $q_{\mathrm{rad}, o}$ is the radiation heat flux from the outer surface of textile to the ambience. $q_{\text {conv }}$ is the convective heat flux from the outer surface of textile. All parameters are calculated with the equations (S4-S11) and Table S1.

\section{Expressions of terms involved in heat transfer model analysis}

$$
\begin{gathered}
q_{\text {rad }, s}=\sigma T_{s}^{4} \quad \text { (S4) } \\
q_{r a d, i}=\varepsilon_{i} \sigma T_{i}^{4} \quad \text { (S5) } \\
q_{\text {rad }, o}=\varepsilon \sigma T_{o}^{4} \text { (indoor) } \quad \text { (S6) } \\
q_{\text {rad }, o}=\varepsilon_{o} \int_{0}^{\frac{\pi}{2}} \pi \sin (2 \theta) \int I_{B B}\left(T_{o}, \lambda\right) d \lambda \quad \text { (outdoor) } \\
q_{\text {cond }, a}=k_{a} \cdot\left(\frac{T_{s}-T_{i}}{t_{a}}\right) \quad(\mathrm{S} 7) \\
q_{\text {conv }}=h \cdot\left(T_{o}-T_{e}\right) \quad(\mathrm{S} 9) \\
q_{a t m}=\varepsilon_{o} \sigma T_{e}^{4} \quad(\text { indoor }) \\
q_{\text {atm }}=\int_{0}^{\frac{\pi}{2}} \pi \sin (2 \theta) \int I_{B B}\left(T_{e}, \lambda\right) \varepsilon(\lambda)\left(1-\tau_{M I R}(\lambda)^{1 / \cos (\theta)}\right) d \lambda \quad \text { (outdoor) }
\end{gathered}
$$

All the input parameters are listed in Table S1. 
Table S1. Parameters used in the analysis of heat transfer model.

\begin{tabular}{|c|c|c|c|}
\hline Symbol & Definition & Value & Unit \\
\hline$k$ & Thermal conductivity & $\begin{array}{l}\text { Textile, } k_{t}=0.035 \\
\text { Air gap, } k_{a}=0.03\end{array}$ & {$\left[\mathrm{~W} \mathrm{~m}^{-1} \mathrm{~K}^{-1}\right]$} \\
\hline$t$ & Thickness & $\begin{array}{l}\text { Textile, } t_{t}=0.5 \\
\text { Air gap, } t_{a}=0.3\end{array}$ & {$[\mathrm{~mm}]$} \\
\hline$T$ & Temperature & $\begin{array}{l}\text { Skin, } T_{s}=307.15 \\
\text { Environment, } T_{e}=298.15 \\
\text { Textile inner surface, } T_{i} \\
\text { Textile inner surface, } T_{o}\end{array}$ & {$[\mathrm{~K}]$} \\
\hline$h$ & $\begin{array}{l}\text { Natural convective heat } \\
\text { transfer coefficient }\end{array}$ & 15 & {$\left[\mathrm{~W} \mathrm{~m}^{-2} \mathrm{~K}^{-1}\right]$} \\
\hline$\sigma$ & $\begin{array}{l}\text { Stefan-Boltzmann } \\
\text { constant }\end{array}$ & $5.67 \times 10^{-8}$ & {$\left[\mathrm{~W} \mathrm{~m}^{-2} \mathrm{~K}^{-4}\right]$} \\
\hline$\varepsilon$ & IR emissivity & $\begin{array}{l}\text { Textile inner surface, } \varepsilon_{i} \\
\text { Textile outer surface, } \varepsilon \\
\text { Cotton, } \varepsilon_{c}=0.88 \\
\text { Skin, } \varepsilon_{s}=1\end{array}$ & Unitless \\
\hline rMIR & IR reflectance & $\begin{array}{l}\text { Textile inner surface, } \\
r_{i, M I R}=1-\varepsilon_{i} \\
\text { Textile outer surface, } \\
r_{o, M I R}=1-\varepsilon_{o}\end{array}$ & unitless \\
\hline$\tau_{M I R}$ & IR transmittance & $\begin{array}{l}\text { Atmosphere, Spectral selective } \\
12\end{array}$ & unitless \\
\hline$\alpha$ & Sunlight absorptivity & $\begin{array}{l}\text { Cotton, } \alpha_{c}=0.03 \\
\text { Skin, } \alpha_{s}=0.57 \\
\text { Textile, } \alpha=1-r_{\text {sun }}-\tau_{\text {sun }}\end{array}$ & unitless \\
\hline$r_{\text {sun }}$ & Sunlight reflectance & $\begin{array}{l}\text { Cotton, } r_{\text {sun }, c}=0.67 \\
\text { Textile, } r_{\text {sun }}\end{array}$ & unitless \\
\hline
\end{tabular}




\begin{tabular}{|l|l|l|l|}
\hline$\tau_{\text {sun }}$ & Sunlight transmittance & $\begin{array}{l}\text { Cotton, } \tau_{\text {sun }, c}=0.3 \\
\text { Other textiles, } \tau_{\text {sun }}=0\end{array}$ & unitless \\
\hline
\end{tabular}

*The convective heat transfer coefficient is derived from the experiments.

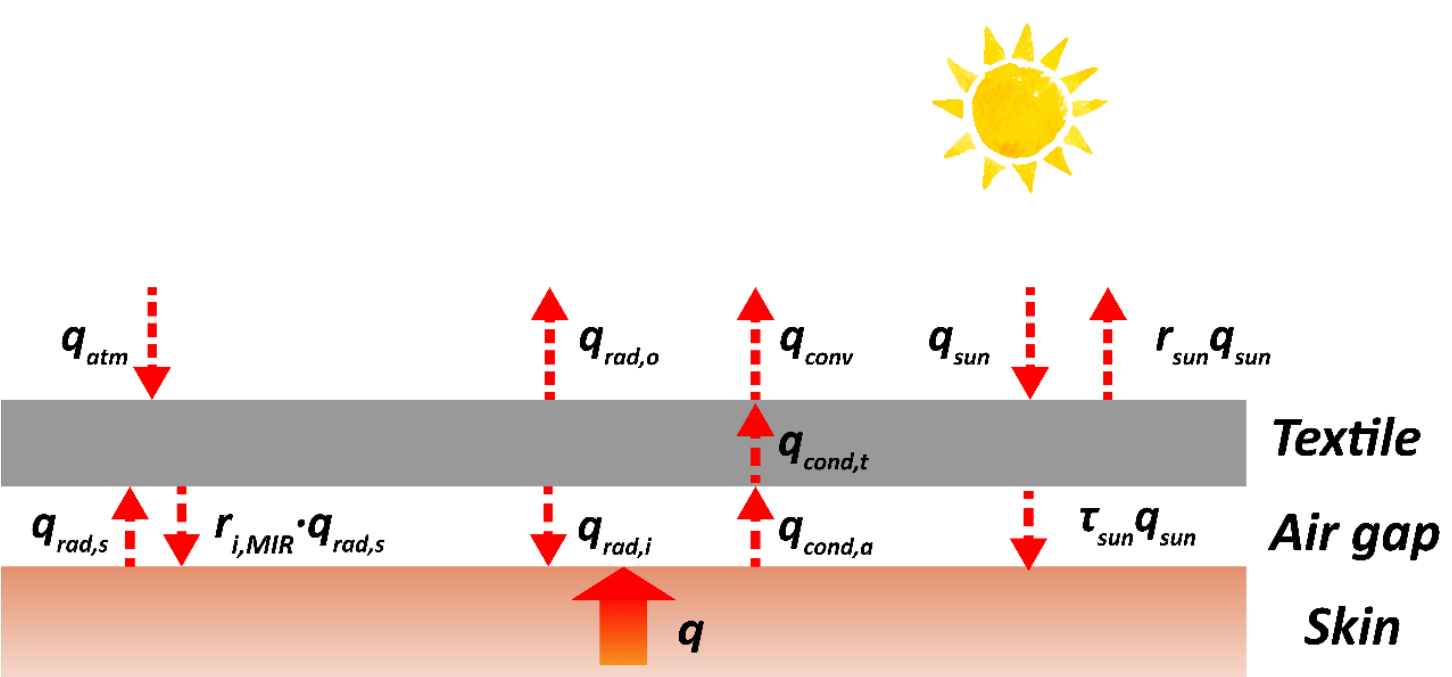

Figure S1. Heat transfer model of textiles. Schematic for one-dimensional steady state heat transfer model.

(a)

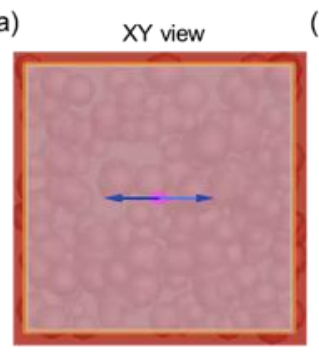

$X Z$ view

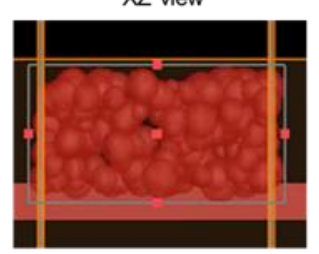

(b)

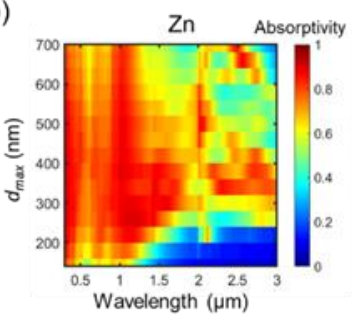

$\mathrm{Zn}$

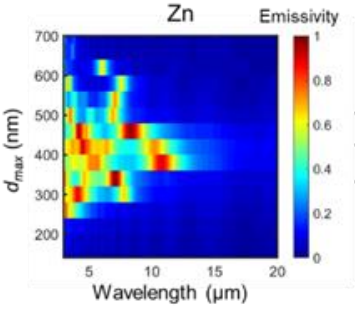

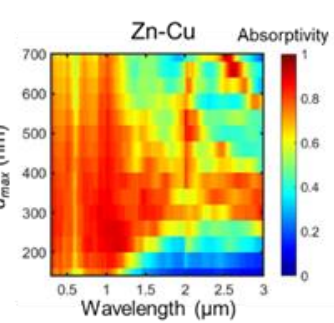

$\mathrm{Zn}-\mathrm{Cu}$

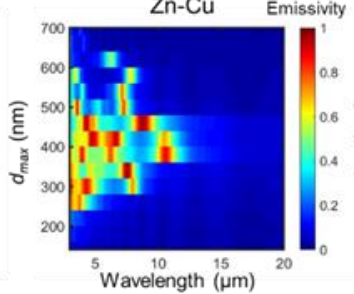

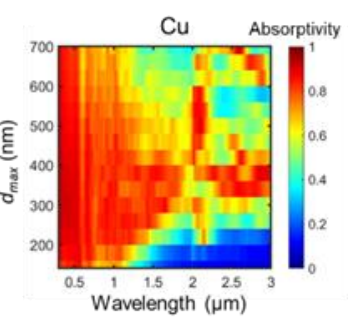

$\mathrm{Cu}$

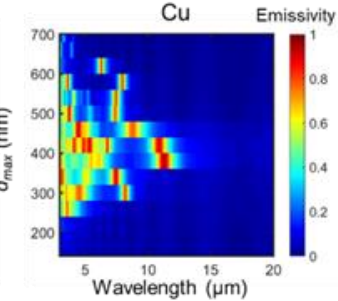

Figure S2. (a) The XY and XZ view of the model in FDTD simulation. (b)The absorptivity and emissivity spectrum of $\mathrm{Zn}$ nanoparticles, $\mathrm{Zn}-\mathrm{Cn}$ nanoparticles with $\mathrm{Cu}$ shell thickness of $10 \mathrm{~nm}$, and $\mathrm{Cu}$ nanoparticles. $\mathrm{d}_{\max }$ is the largest particle diameter for the Gaussian distributed nanoparticles. 

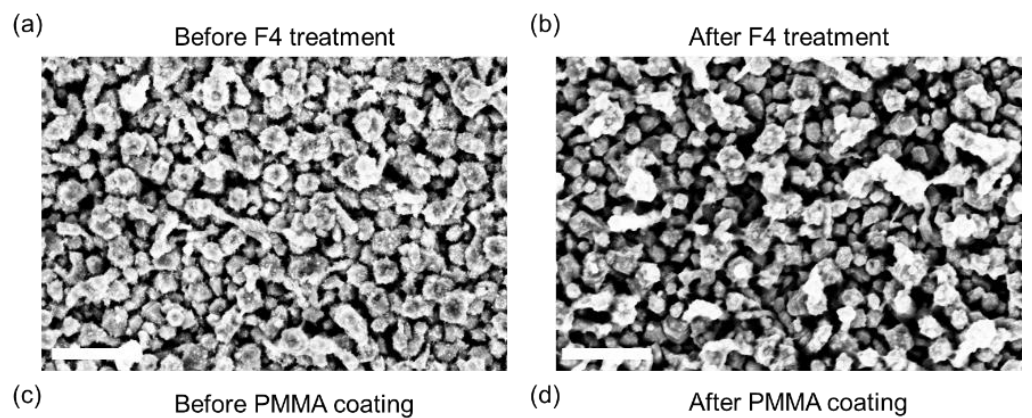

(c)

Before PMMA coating

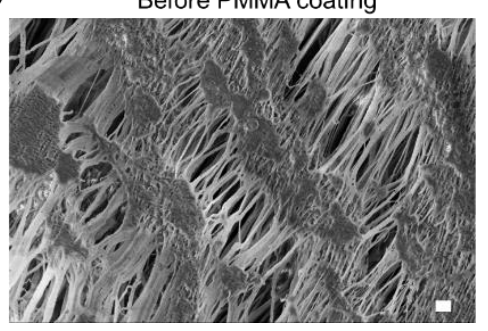

(d)

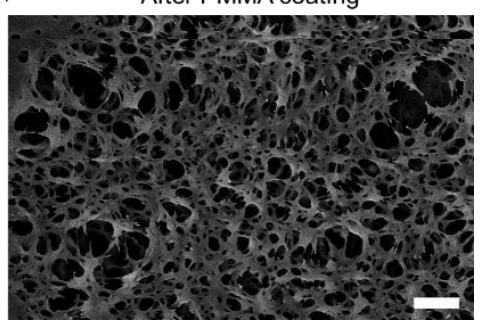

Figure S3. The SEM image of the $\mathrm{Zn}-\mathrm{Cu}$ side (a) before and (b) after F4 treatment and SEM image of the ePTFE (c) before and (d) after PMMA coating. The white scale bar is $1 \mu \mathrm{m}$.

Table S2. The thickness of each layer

\begin{tabular}{|l|l|l|l|l|l|l|}
\hline & $\begin{array}{l}\text { Zn-Cu } \\
\text { coating }\end{array}$ & nPE & Al & ePTFE & PMMA & F4 \\
\hline Thickness & $800 \mathrm{~nm}$ & $\sim 16 \mu \mathrm{m}$ & $90 \mathrm{~nm}$ & $\sim 300 \mu \mathrm{m}$ & $\sim 20 \mu \mathrm{m}$ & Negligible \\
\hline
\end{tabular}


(a)

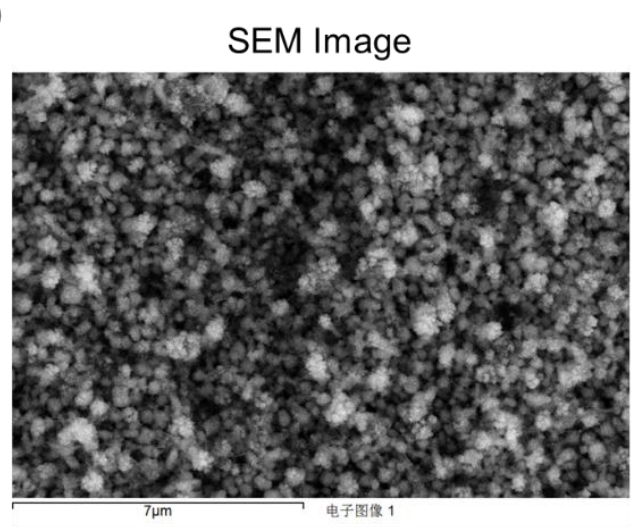

(b)

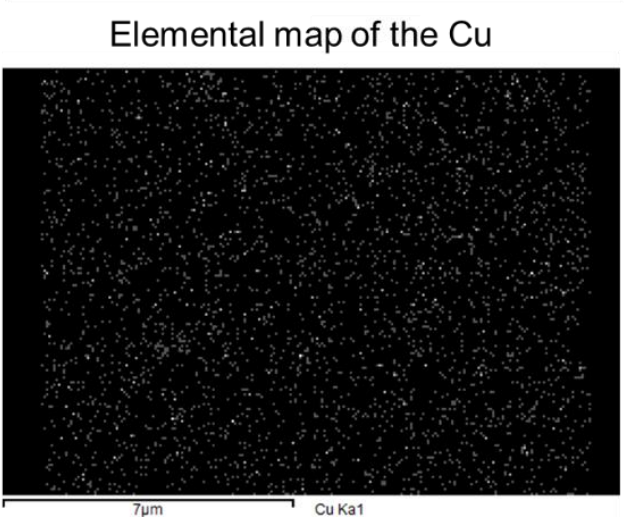

(c) Elemental map of the $\mathrm{Zn}$

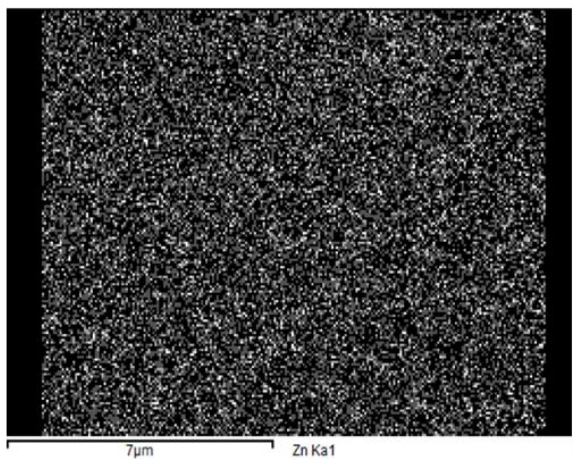

(d)

Energy-dispersive X-ray spectra

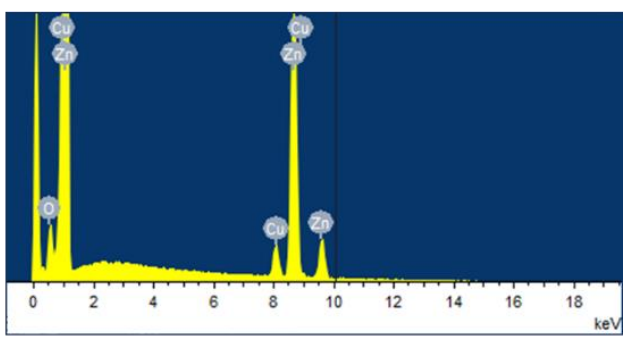

Figure S4. (a) SEM image of zinc $(\mathrm{Zn})$ and copper $(\mathrm{Cu})$ nanoparticle solar absorptive coating (30 s immersion time in $5 \mathrm{mM} \mathrm{CuSO}_{4}$ solution). (b), (c) Elemental maps of the $\mathrm{Cu}$ (b) and $\mathrm{Zn}$ (c) nanoparticles in panel (a). (d) Energy-dispersive X-ray spectra of the nanoparticle s in panel (a). The scale bar represents a length of $7 \mu \mathrm{m}$.

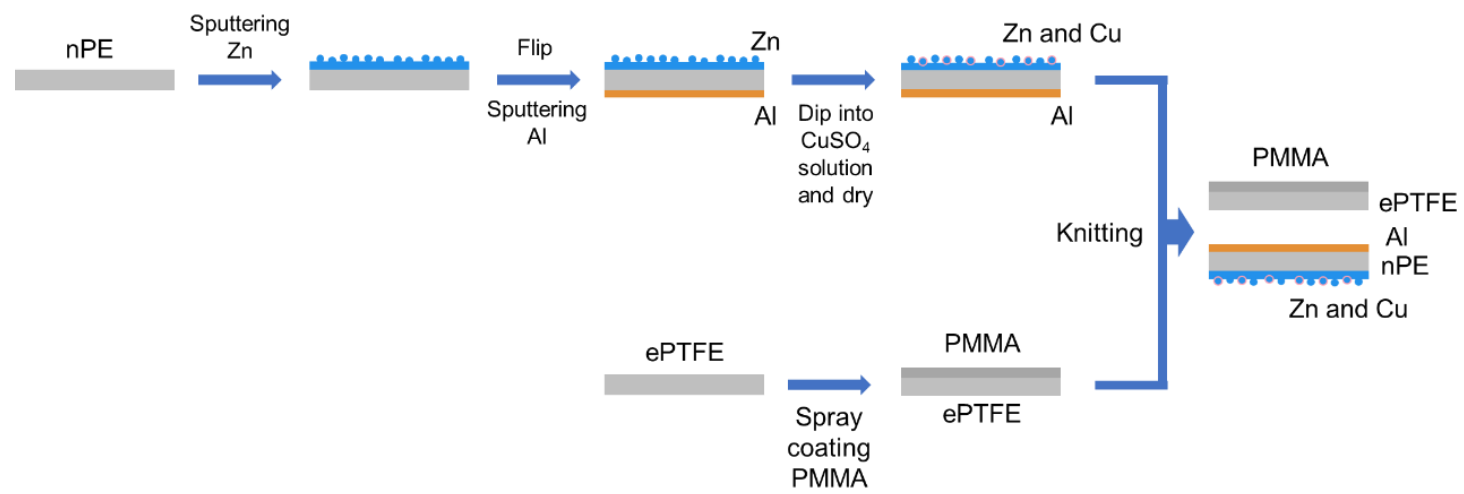

Figure S5. Fabrication process of the Janus textile. 
(a)

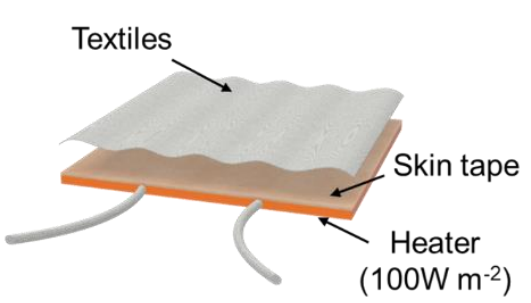

(b)

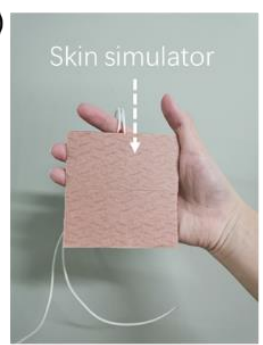

(d)

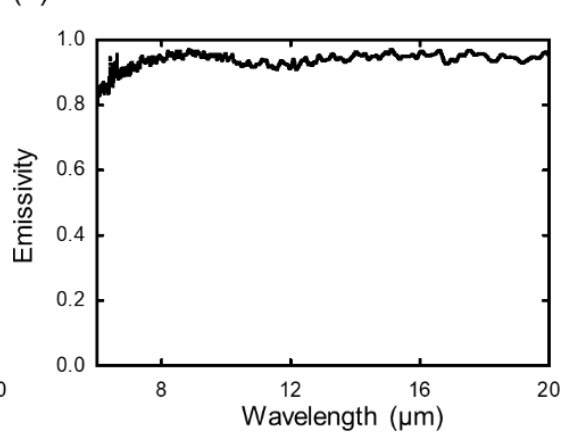

Figure S6. (a) Schematic and (b) Optical image of the skin simulator. (c) Sunlight reflectivity and (d) mid-infrared emissivity spectrum of the skin simulator.

(a)

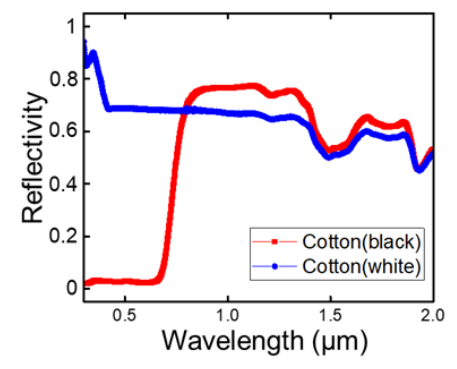

(b)

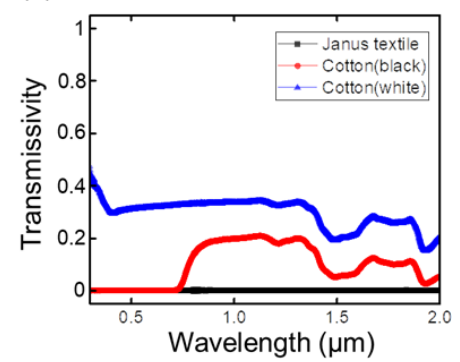

(c)

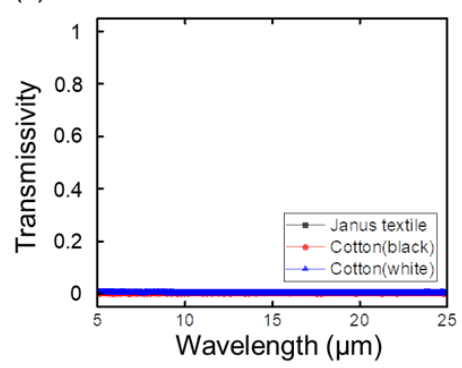

Figure S7. (a)Sunlight reflectivity of the black and white cotton. Transmissivity of the textiles for (b) sunlight and (c) thermal wavelength ranges. The Janus textile in this work is opaque for all wavelengths of interest. 
(a)

(c)

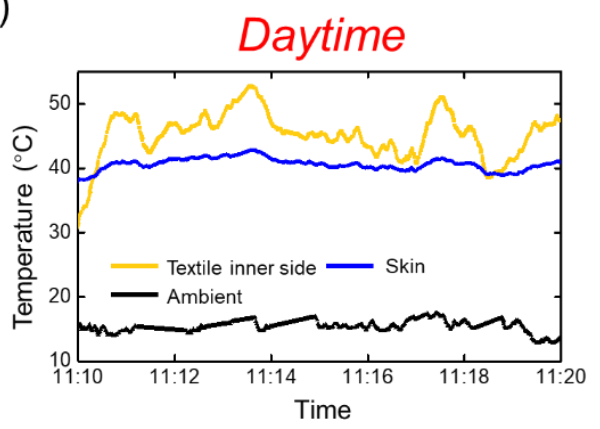

Janus textile (heating mode)/skin (b)
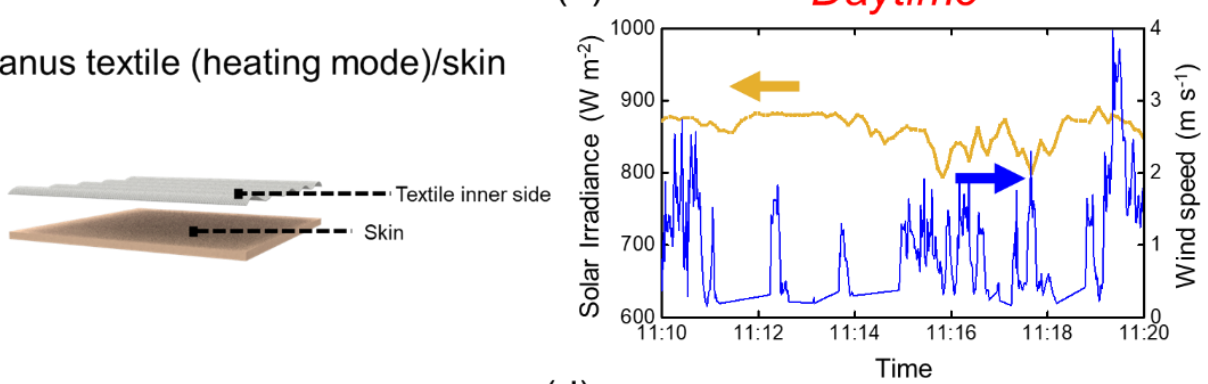

(d)

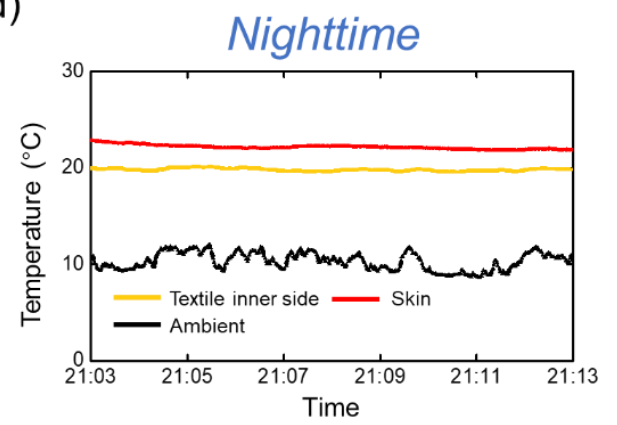

Figure S8. (a) Schematic for the textile inner surface and skin temperature measurement. (b) Measured solar irradiance and wind speed during daytime test. (c) Daytime measured textile inner surface and skin temperature. (d) Nighttime measured textile inner surface and skin temperature.

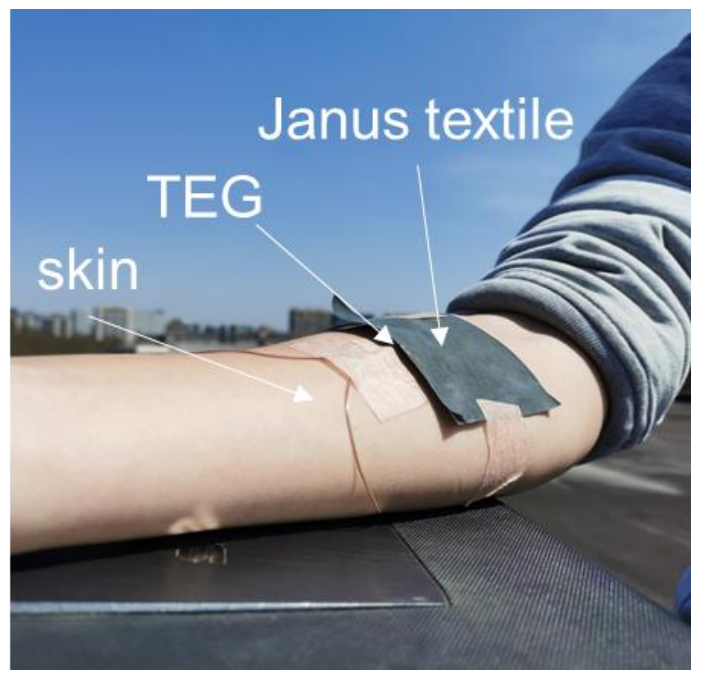

Figure S9. Optical image for thermoelectricity generation measurement. 


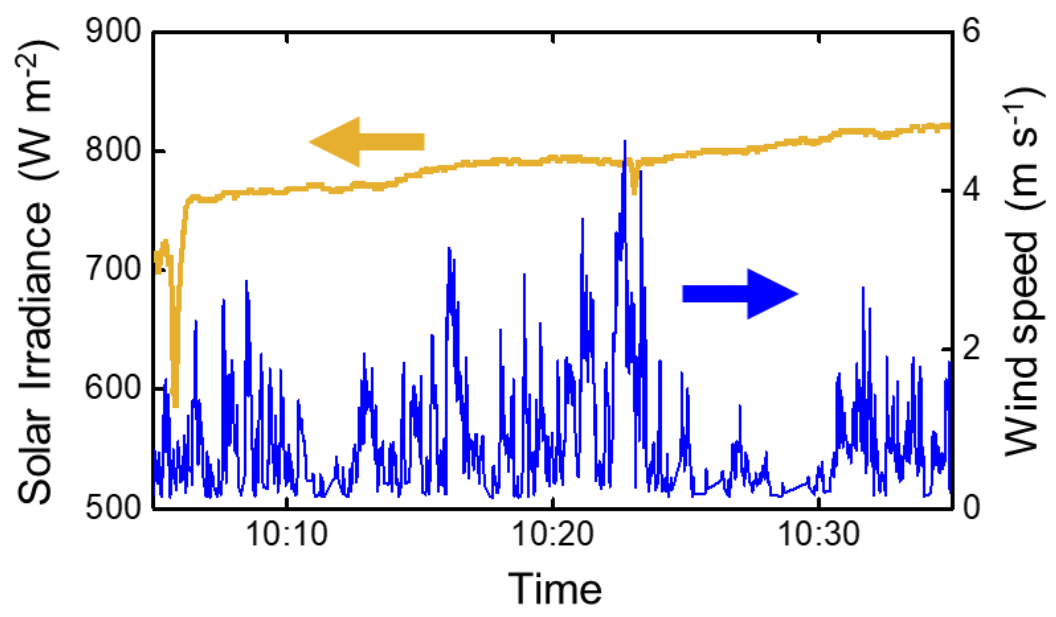

Figure S10. The solar irradiance power density of the daytime outdoor thermoelectricity generation measurement.

(a)

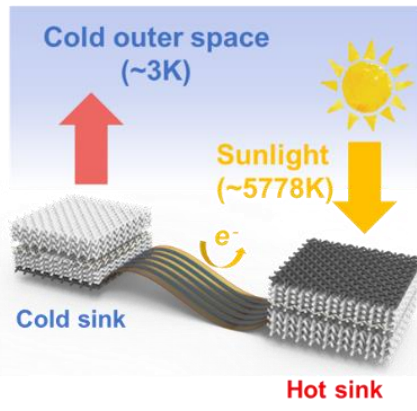

(b)

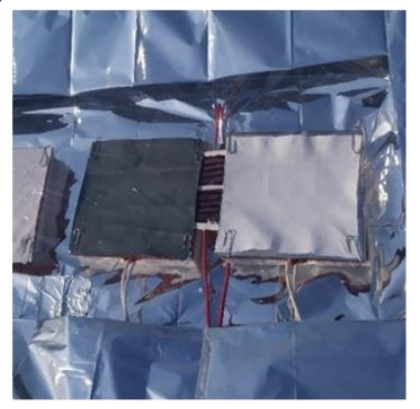

(c)

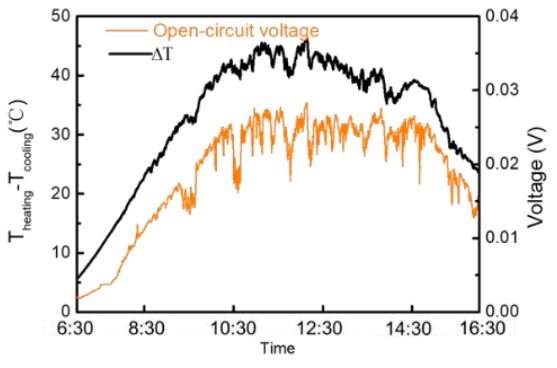

Figure S11. (a) Schematic and (b) the optical image of a homemade wearable thermal electricity generation device. The Janus textiles in different modes are attached to the two ends of a home-made wearable thermoelectric modules (10 pairs of AZO/PEDOT:PSS as n-type and PEDOT:PSS as p-type). (c) The measured temperature difference of the Janus textile working in different modes and corresponding output power voltage. 
(a)

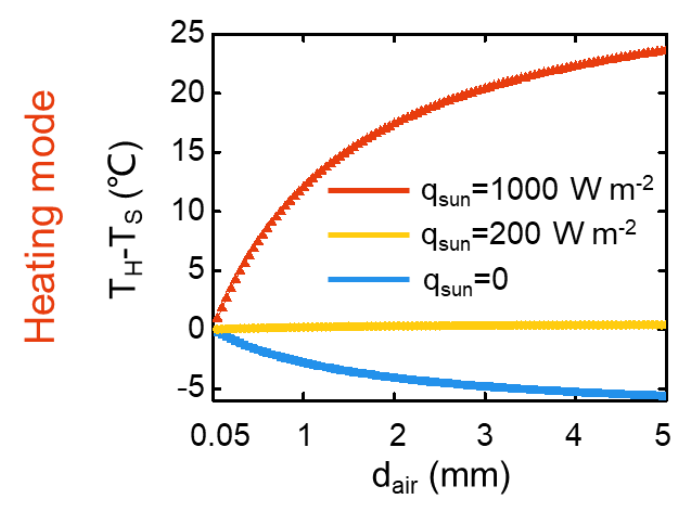

(c)

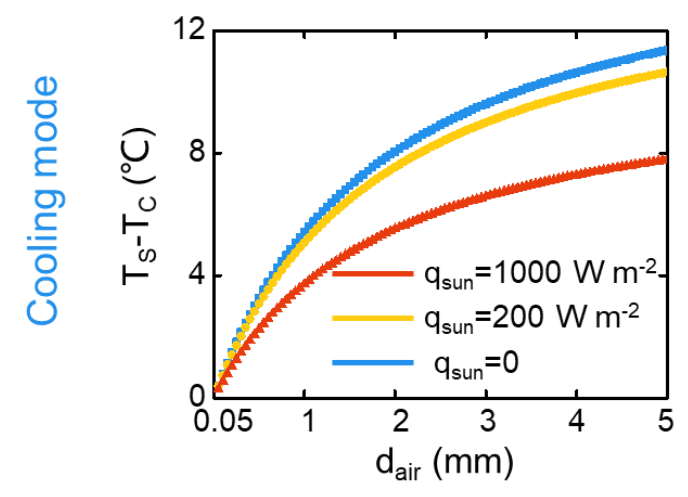

(b)

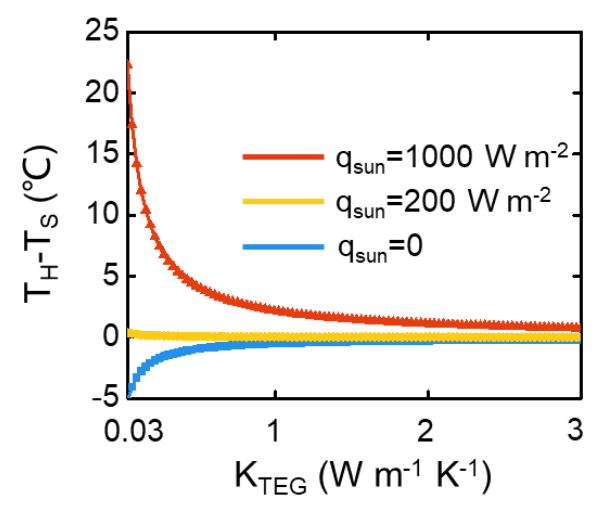

(d)

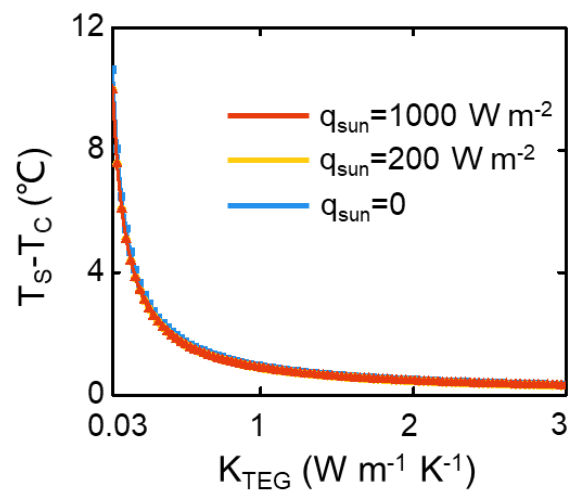

Figure S12. (a) Calculated temperature difference of the hot side (Janus textile in heating mode, $\mathrm{T}_{\mathrm{H}}$ ) and the skin $\left(\mathrm{T}_{\mathrm{S}}\right)$ for air gaps of different thicknesses or (b) thermocouple modules (TEG) of different thermal conductivities. (c) Calculated temperature difference of the cold side (Janus textile in cooling mode, Tc) and the skin (Ts) for air gaps of different thicknesses or (d) thermocouple modules (TEG) of different thermal conductivities. $q_{\text {sun }}$ represents the solar irradiance power density. Large air gap (or TEG thickness) or low thermal conductivity TEG will help increase the temperature difference to enhance output power. 

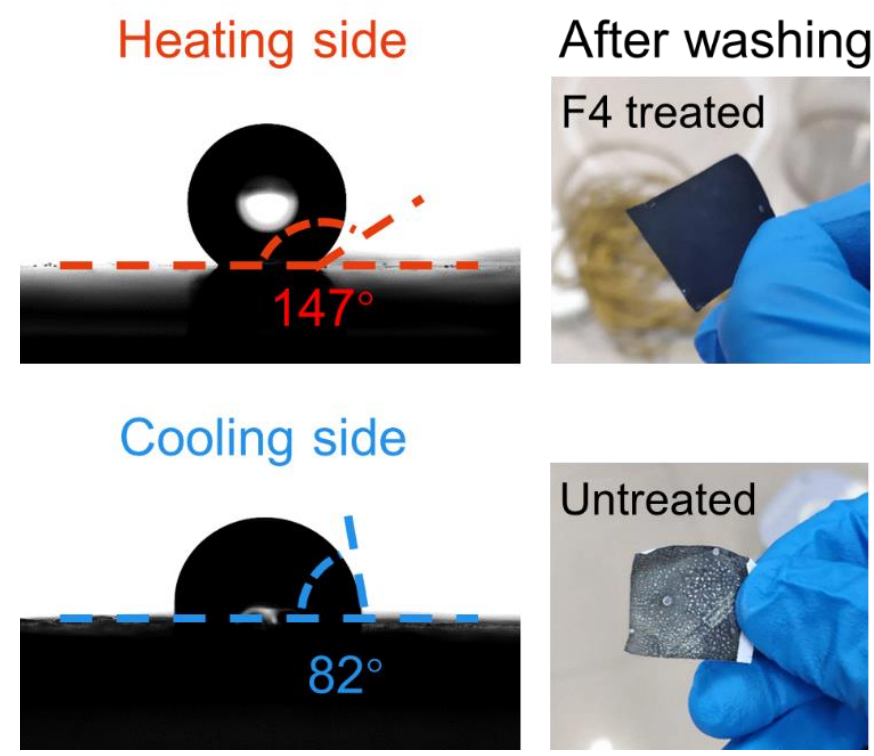

Figure S13. The water contact angle of the two sides of the Janus textile and the optical image of the F4 treated and untreated Janus textile after washing.

(a)

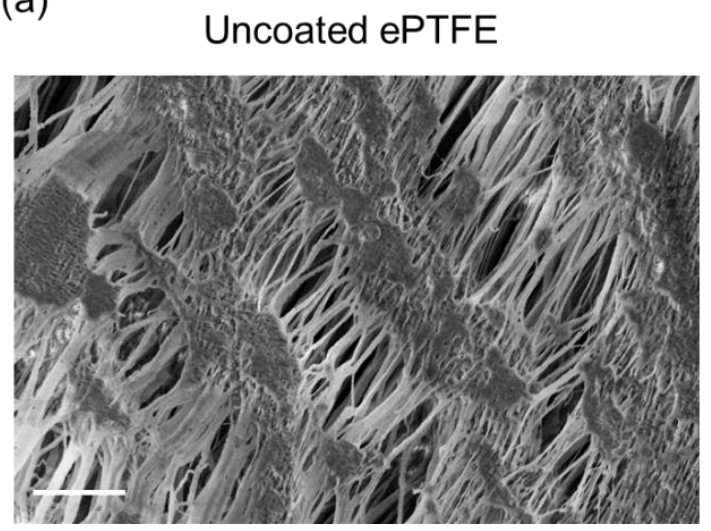

(b)

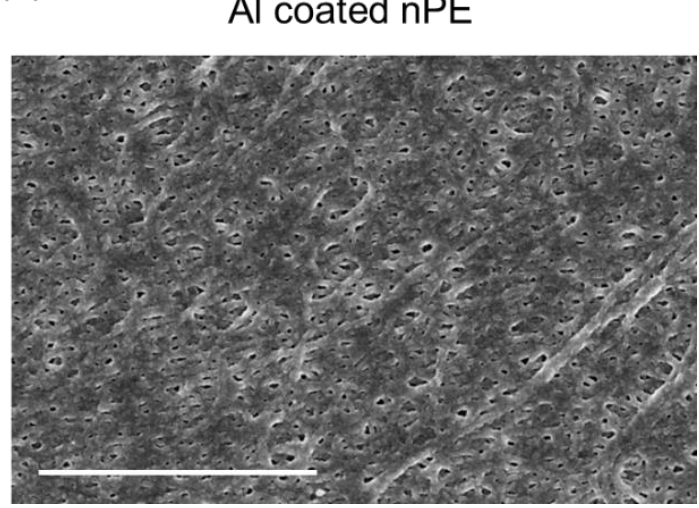

Figure S14. (a) SEM image of the $300 \mu \mathrm{m}$ uncoated ePTFE textile used as a part of daytime radiative cooling side of the Janus textile (a thin gold film was sputtered onto the textile to enhance the conductivity without changing the morphology). (b) SEM image of the aluminum (Al) coated nanoporous PE (nPE). The well distributed nanopores allows water vapor to transmit. The scale bar represents a length of $4 \mu \mathrm{m}$. 